\title{
Identification and Characterisation of Alternative Splice Variants of Hoxb9 and Their Correlation with Melanogenesis in the Black-Boned Chicken
}

http://dx.doi.org/10.1590/1806-9061-2018-0904

Original Article

\section{-Author(s)}

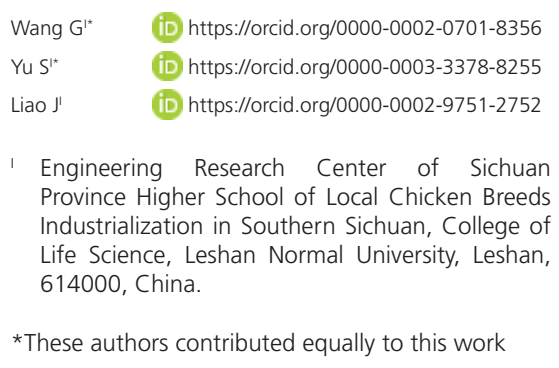

\section{-Mail Address}

Corresponding author e-mail address Shigang Yu

Leshan Normal University Ringgold standard institution, Nº 778, Binhe Road, shizhong distinct, Leshan, Sichuan, Leshan, Sichuan 614000 - China.

Phone: +86-833-2277896

Email: shigang_yu@163.com

\section{- Keywords}

Homeobox B9 gene; Alternative splicing variant; Melanin; Chicken.

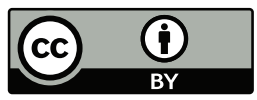

Submitted: 19/September/2018

Approved: 06/January/2019

\section{ABSTRACT}

Homeobox B9 (HOXB9) gene has been demonstrated to be associated with melanogenesis in chicken plumage by high-throughput RNA sequencing. In this study, we cloned and characterised HOXB9 in black-boned chickens. Two alternative splice variants (HOXB9-1 and HOXB9-2) were identified in chicken feather bulbs. Expression analysis of HOXB9 in 11 different chicken tissues by RT-PCR indicated that the two transcripts were only expressed in the kidney, abdominal fat, feather bulbs, skin, and small intestine. No HOXB9-1 or HOXB92 transcripts were detected in the breast muscle or the ovary. The two HOXB9 variants were expressed at significantly different levels in black feather bulbs and white feather bulbs $(p<0.01)$, and in black skin compared with white skin $(p<0.01)$. The results indicate that HOXB9-1 and HOXB9-2 may be involved in melanin formation in the plumage and skin. The expression of HOXB9-2 was higher in white than in black muscles $(p<0.05)$, HOXB9-2 may play a role in muscle melanogenesis. Our results suggest that HOXB9, which is partially regulated by alternative splicing, may be involved in the process of melanogenesis in the black-boned chicken.

\section{INTRODUCTION}

Black-boned chickens demonstrate intense pigmentation (hyperpigmentation or fibromelanosis) of their tissues, including the skin, muscle, feathers, claws, gonads, periosteum, and mesentery (Faraco et al., 2001; Dorshorst et al., 2011; Yu et al., 2017). Feather, skin, and muscle pigmentation phenotypes are important economic traits in black-boned chicken production, as chickens with black feathers, skin, and muscles are generally considered to have superior nutritional and medicinal properties (Tian et al., 2007). Melanin-based pigmentation is a major component of chicken feather, skin, and muscle colouration that is often under strong genetic control (Bourgeois et al., 2016). Many signalling pathways and numerous genes are involved in melanin biosynthesis, transport, transfer, and deposition (Cieslak et al., 2011; Zhang J et al., 2015; Zhang XD et al., 2015). To date, more than 150 body colour-associated genes have been found to play wellknown roles in pigmentation (Cieslak et al., 2011). Therefore, further exploration of other candidate genes using different techniques may be required to elucidate the complex molecular mechanisms of melanin pigmentation.

In a previous study, we identified the expression of the Homeobox B9 (HOXB9) gene associated withblack-boned chicken plumage melanogenesis, based on transcriptome analysis of black and white chicken feather bulbs using high-throughput RNA sequencing (Yu et al., 2018). HOXB9 encodes nuclear protein functions as a transcription 
factor involved in cell proliferation and differentiation (Seki et al., 2012). HOXB9 was also identified as a Wnt target gene (Nguyen et al.,2009). Wnt signalling is essential for the complex organogenesis of the skin and its appendages, such as hairs, feathers, and scales (Widelitz, 2008). Moreover, Wnt/ $\beta$-catenin signalling plays a key role in melanocyte development, proliferation, and differentiation (Yamaguchi, 2004; Yamaguchi et al., 2007; Cho et al., 2009).

In order to further elucidate the role of the HOXB9 gene in melanin formation and deposition, we cloned the HOXB9 gene and identified two alternative splice variants in black-boned chickens, andanalysedthe relationship between the expression of these alternative splice variants and melanin synthesis in different tissues. Our results provide new insights to further the understanding ofthe molecular mechanisms of melanogenesis in the black-boned chicken.

\section{MATERIAL AND METHODS}

\section{Ethics statement}

All experiments involving animals were approved by the Animal Care and Use Committee of Leshan Normal University, China. The procedures were performed in accordance with the approved guidelines and regulations of the regional Animal Ethics Committee.

\section{Birds and sample collection}

Eighteen52-week-oldfemale Muchuanblack-boned chickens reared in the breeding farm of Muchuan County Black Phoenix Black-boned Chicken Industry Co. Ltd. were evaluated in this study. All chickens were reared under the same conditions. Birds were fed and managed by the farm employees according to the poultry company's practices.

Birds were killed by exsanguination for the collection of skin, feather bulbs (black), kidney, small intestine, abdominal fat, liver, gizzard, breast muscle, heart, comb, and ovary tissues. These tissues were frozen in liquid nitrogen immediately after collection and stored at $-80^{\circ} \mathrm{C}$ prior to RNA extraction.

Feather bulbs were collected from three chickens with black (BF group: BF1, BF2, and BF3) or white (WF group: WF1, WF2, and WF3) dorsal plumage; muscle samples were obtained from three chickens with black (BM group: BM1, BM2, and BM3) or white (WM group: WM1, WM2, and WM3) breast muscle; and skin samples were obtained from three chickens with black (BS group: BS1, BS2, and BS3) or white (WS group: WS1, WS2, and WS3) skin (Figure 1).

\section{RNA extraction and reverse transcription}

Total RNA from each sample was isolated using Invitrogen TRIzol reagent (Life Technologies, Carlsbad, (A, USA) in accordance with the manufacturer's instructions. RNA degradation and contamination were monitored on $1 \%$ agarose gels. Reverse transcription was performed using the ProtoScript First Strand cDNA Synthesis Kit (NEB, Beijing, China), with the reaction system consisting of $1 \mu \mathrm{g}$ total RNA, 2 $\mu \mathrm{L} d(\mathrm{~T}) 23 \mathrm{VN}(50 \mathrm{uM}), 10 \mu \mathrm{L}$ M-Mulv reaction mix, $2 \mu \mathrm{L}$ M-Mulv enzyme mix, and diethylpyrocarbonate (DEPC)-treated water to reach a final reaction volume of $20 \mathrm{uL}$. The reactions were incubated at $42^{\circ} \mathrm{C}$ for $1 \mathrm{~h}$ and terminated by heating at $80^{\circ} \mathrm{C}$ for 5 min. Reaction products were diluted to $100 \mu \mathrm{L}$ with DEPC-treated water for $P C R$, and stored at $-20^{\circ} \mathrm{C}$.
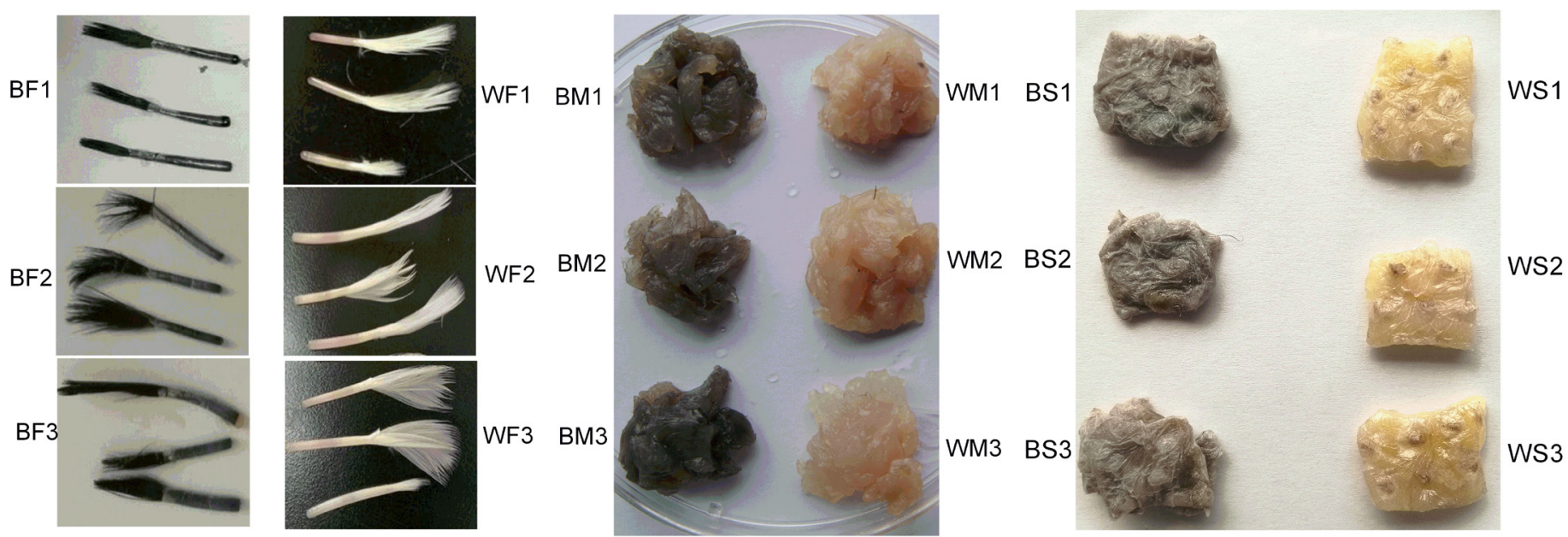

Figure 1 - Phenotypic characterisation. (a) Feather bulb colours of the two types of feather bulbs: black feather bulbs (BF) and white feather bulbs (WF). (b) Meat colour of the two types of breast muscle: black breast muscle (BM) and white breast muscle (WM). (c) Skin colour of the two types of skin: black skin (BS) and white skin (WS). 


\section{Molecular cloning of chicken HOXB9 and its alternative splice variants}

Primer $\mathrm{H}$ (Table 1) was designed to amplify the coding sequence of chicken HOXB9 using the CDNA sequence retrieved from the NCBI database (XM_015299445.1). The PCR reaction, performed using $2 \times$ EasyPruf PCR SuperMix (TransGen Biotech, Beijing, China) according to the manufacturer's instructions, generated a fragment of about $750 \mathrm{bp}$. The PCR products were verified on $1.5 \%$ agarose gels at constant $120 \vee$ for 30min, and the target bands were excised under UV light and purified using the E.Z.N.A. Gel Extraction Kit (Omega Bio-Tek, Inc., Norcross, GA, USA) according to the manufacturer's instructions. The purified products were subcloned in the pEASY-T3 vector (TransGen Biotech, Beijing, China) according to the manufacturer's instructions. The positive clones with different lengths of chicken HOXB9 cDNA sequence were sequenced by TsingKe Biological Technology (Chengdu, China). Finally, two alternative splice variants of chicken HOXB9 were identified by sequencing.

Table 1 - Primers used in this study.

\begin{tabular}{llcc}
\hline Name & Sequence $\left(5^{\prime}-3^{\prime}\right)$ & $\begin{array}{c}\text { Annealing } \\
\text { temperature }\end{array}$ & $\begin{array}{c}\text { Product } \\
\text { length }\end{array}$ \\
\hline H1 & F: ATGTCCATTTCTGGGACTCTTAGC & $60^{\circ} \mathrm{C}$ & $744 / 822 \mathrm{bp}$ \\
R:TCACTCCTTGCCCTGCTCCT & & $189 \mathrm{bp}$ \\
HS-2 & F: GATCTGTTAAGGCAGAGCCG & $60^{\circ} \mathrm{C}$ & \\
& R: CAGCTGGGTTGGTTGATCTG & & \\
H2 $(\beta-$ Actin) & F: AGGCAAGGGAAGGGATCGTT & $60^{\circ} \mathrm{C}$ & $138 \mathrm{bp}$ \\
& R: CACGCTCCTGGAAGATAG & & \\
\hline
\end{tabular}

Tissue distribution pattern of HOXB9 gene expression

Quantitative real-time PCR (qRT-PCR) was used to determine the mRNA levels of the two alternative splice variants of HOXB9 in the 11 tissues described above. The volume of each qRT-PCR reaction was $20 \mu \mathrm{L}$, containing $1 \mu \mathrm{L}$ reverse transcription reaction mix, $0.4 \mu \mathrm{L}$ of each primer $(10 \mu \mathrm{mol} / \mathrm{L}), 10 \mu \mathrm{L}$ SYBR Green Master Mix (Vazyme, Nanjing, China), $0.4 \mu \mathrm{L}$ $50 \times$ ROX Reference Dye, and $8.2 \mu \mathrm{L} \mathrm{ddH}_{2} \mathrm{O}$. qRT-PCR was performed using a StepOne Plus Real-Time PCR system (Applied Biosystems, Foster City, CA, USA) as follows: $95^{\circ} \mathrm{C}$ for $5 \mathrm{~min}$ followed by 40 cycles of $0 \mathrm{~s}$ at $95^{\circ} \mathrm{C}, 30 \mathrm{~s}$ at $60^{\circ} \mathrm{C}$, and melt curve analysis from $60^{\circ} \mathrm{C}$ to $95^{\circ} \mathrm{C}$. All reactions were performed in triplicate. To normalise gene expression, the $\beta$-actin gene was selected as internal control. Relative gene expression levels were calculated using the comparative $\mathrm{Ct}(\Delta \Delta \mathrm{Ct})$ value method. The specific primers (HS-1 and HS-2, Table 1) for the two alternative splice variants (HOXB91 and HOXB9-2) were designed based on the unique sequence of the splicing boundary of each variant.

\section{Expression patterns of theHOXB9 gene in albino and black samples}

To investigate the functions of the two alternative splice variants of HOXB9 in melanin biosynthesis in different tissues (feather bulbs, skin, and breast muscle), qRT-PCR was performed to determine the differential expression levels of HOXB9 between group $B F$ and WF, group BM and WM, and group BS and WS. The gene-specific primers used are shown in Table 1. Statistical analyses were performed using Student's $t$-test for independent samples, $p<0.05$ was considered significant.

\section{Sequence analysis of the chicken HOXB9 gene}

The obtained cDNA sequences were matched using DNAMAN software (version 8.0.8.789). BLAST (http://www.ncbi.nlm.nih.gov/BLAST) was used to identify homologous sequences in the NCBI database. The HOXB9 gene open reading frame (ORF) was predicted using the ORF Finder at NCBI (https://www.ncbi.nlm.nih.gov/orffinder). Chromas software (version 2.23; Technelysium, Tewantin, Australia) was employed to examine the sequence chromatograms. Related sequences were obtained from the Ensembl database (http:// www.ensembl.org) and the GenBank database (http://www.ncbi.nlm.nih.gov/genbank).

\section{RESULTS}

\section{cDNA sequence analysis of the chicken HOXB9 gene}

The chicken HOXB9 gene was amplified by RT-PCR using the primer $\mathrm{H} 1$. Two PCR products of different lengths were obtained from black feather bulbs (Figure 2), suggesting that the chicken HOXB9 gene may have two alternative splice variants, HOXB9-1 (GenBank accession number: MH048960) and HOXB9-2 (GenBank accession number: MH048961).

The HOXB9-1 transcript contains an ORF of 741 base pairs (bp) and encodes a protein of 247 amino acids (aa). The HOXB9-2 transcript contains an819-bp ORF, which is generated due to a 78-bp fragment inserted in the HOXB9-1 transcript, and encodes a 273-aa polypeptide. 


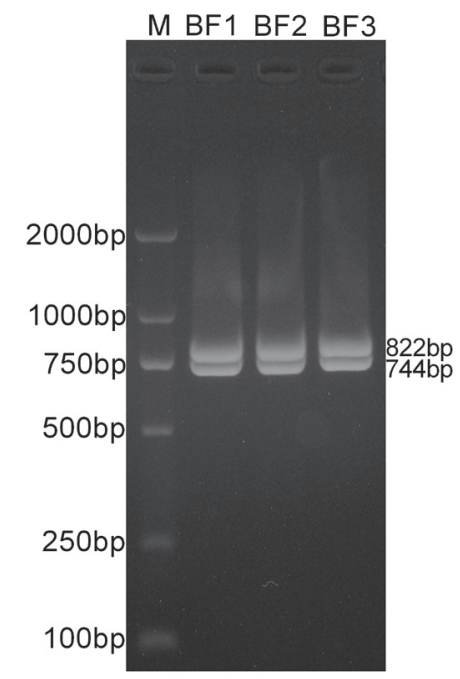

Figure 2 - Amplified products of the chicken HOXB9 gene. Lane M, D2000 DNA Marker.

\section{Structural analysis of the chicken HOXB9 gene}

HOXB9-1 has a standard gene structure and includes two exons and one intron, which have nucleotide sizes of 508, 336, and 3,254 bp, respectively. The total length of the HOXB9-2 coding region is $822 \mathrm{bp}$, with a 78-bp insert at the $3^{\prime}$ end of exon 1, which results in an insert of 26 amino acid residues (Figure 3) compared with the normal HOXB9 gene. The sequence matching and alignment analysis revealed that the 78-bp insert was a partial sequence of the intron; thus, the type of alternative splicing is the partial retention of an intron in the mature transcript (Figure 3).

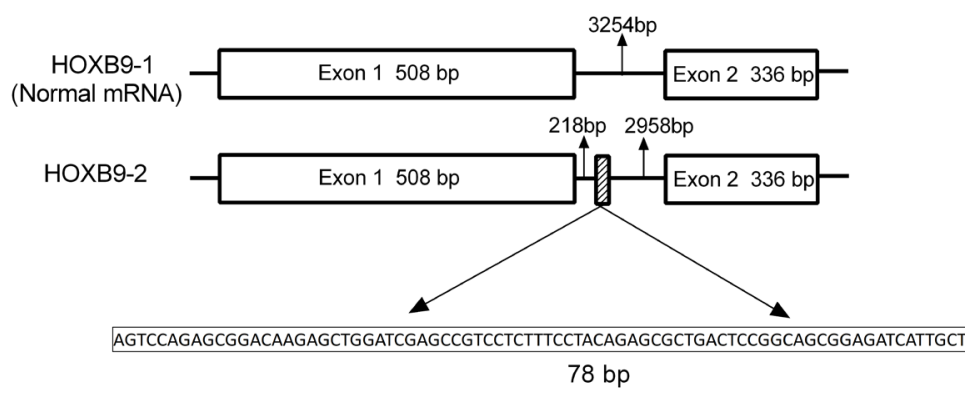

Figure 3 - Schematic diagrams of the structures of alternative splice variants of the HOXB9 gene. Exons are indicated by rectangles, whereas introns are indicated by black lines. The box containing diagonal lines indicates that the variant includes the partial sequence of an intron.

\section{Tissue distributions of two HOXB9 transcripts: HOXB9-1 and HOXB9-2}

The mRNA levels of HOXB9-1 and HOXB9-2 in 11 tissues were evaluated by qRT-PCR. Neither transcript of HOXB9 was expressed in the liver, gizzard, heart, or comb tissues. The highest expression levels of HOXB9-1 and HOXB9-2 were detected in the kidney, abdominal fat, feather bulbs, skin, and small intestine tissues (Figure 4). HOXB9-1 and HOXB9-2 were weakly expressed in the ovary and breast muscle tissues, respectively. The mRNA level of HOXB9-2 was significantly higher than that of HOXB9-1 in skin $(p<0.01)$, small intestine $(p<0.001)$, and abdominal fat $(p<0.001)$. No HOXB9-1 or HOXB9-2 transcripts were detected in the breast muscle and ovary, respectively.

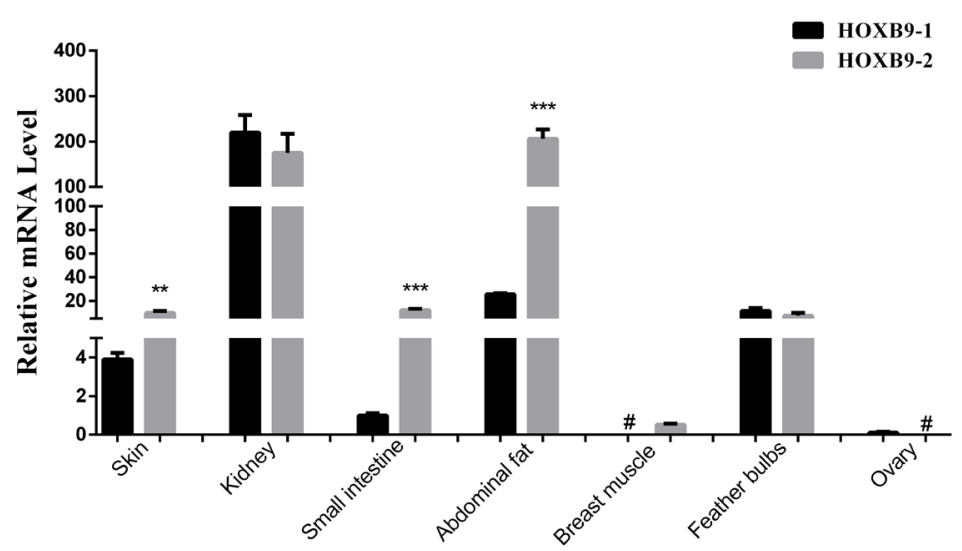

Figure 4 - The mRNA expression profiles of HOXB9-1 and HOXB9-2 in various tissues of the black-bonedchicken. Expression levels of HOXB9-1 and HOXB9-2 within the same tissues were compared using $t$-test, and significant differences are indicated with asterisks ( $\left.{ }^{* *} p<0.01,{ }^{* * *} p<0.001\right)$. \# indicates that no HOXB9 mRNA was detected in the tissue. The mRNA level in small intestine tissue was defined as 1.0.

\section{Functional characterisation of the two HOXB9 variants in melanogenesis in different tissues}

The qRT-PCR analysis revealed that the mRNA levels of HOXB9-1 and HOXB9-2 were higher in the WF group than in the BF group (115.27-fold, $p<0.001$, and 103.60-fold, $p<0.01$ ) (Figure 5a). The mRNA levels of HOXB9-1 and HOXB9-2 were 22.49- and 67.71fold higher in the WS group than in the BS group, respectively $(p<0.01)$ (Figure $5 c)$. The mRNA level of HOXB9-2 was significantly higher than that of HOXB91 in breast muscle $(p<0.05)$ (Figure $5 b)$.

\section{DISCUSSION}

Numerous studies have investigated pigmentation in animals (mammals, birds, and fish). A large number of genes, such as the genes for tyrosinase (TYR) (Chang et al., 2006; Yu et al., 2017), endothelin 3 (EDN3) (Dorshorst et al., 2011), agouti-signalling protein (ASIP)(Voisey \& Van 2002; Zhang J et al., 2015), premelanosome protein (PMEL17) (Kerje et al., 2004), and the Rab family (RAB27A) (Hume et al., 2007), has been reported in different signalling pathways involved in melanin synthesis, pigment type switching, melanosome formation, and pigment transport, transfer, and deposition. However, the genetic mechanisms influencing melanin-based colouration 
a

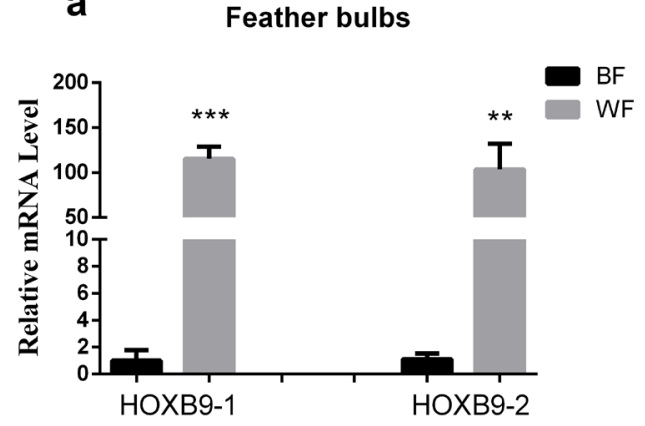

b
Breast muscle

\section{C}

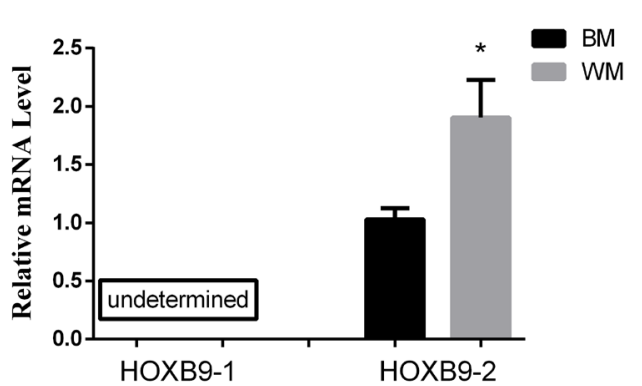

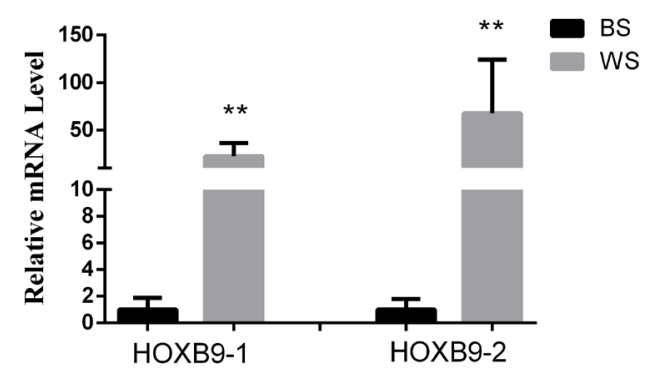

Skin

Figure 5 - HOXB9 mRNA expression levels in various white and black tissue samples from black-bonedchickens. The relative mRNA expression of the two variants were compared between white and black (a) feather bulbs, (b) breast muscle, and (c) skin. ${ }^{*} p<0.05,{ }^{* *} p<0.01,{ }^{* * *} p<0.001$.

remain poorly characterised. The melanogenesis in the black-boned chicken provides an excellent system for studying the molecular mechanisms of melanin pigmentation. Our previous study found that the HOXB9 gene was associated with melanin formation in plumage, and was identified asa pigmentation gene in the chicken (Yu et al., 2018). However, the structure of chicken HOXB9 and its role in melanogenesis, have not yet been elucidated.

In this study, we cloned and characterised HOXB9 in the Muchuan black-boned chicken. Two alternative splice variants were identified in black feather bulbs. Alternative splicing (AS) is a post-transcriptional process that regulates gene expression by increasing protein complexity and modulation of mRNA transcript levels. AS occurs in vertebrate, invertebrate, plant, and fungal genomes (Cheah et al., 2007; Mcguire et al., 2008; Simpson et al., 2010; Mudge et al., 2011).In humans, transcripts from $95 \%$ of multi-exon genes undergo AS (Pan et al., 2008), whereas approximately 35\% of genes undergo AS in Arabidopsis and rice (Simpson et al., 2010). AS events fall into five well-known types of AS patterns, including alternative $5^{\prime}$ and $3^{\prime}$ splice site selection, intron retention, exon skipping, and mutually exclusive exon splicing (Lu et al., 2015). Our study showed partial intron retention in HOXB9-2.

Many alternatively spliced isoforms are known to have tissue-preferential expression patterns, and differential AS has been demonstrated to play important roles in various biological processes (Thatcher et al., 2014). In this study, we found that the two alternative splicing variants of HOXB9 showed tissue-specific expression patterns, and no expression was found in the liver, muscular stomach, heart, and comb tissues. The higher HOXB9 mRNA level in the skin tissue allows for its functional role in skin development. In addition, the mRNA level of HOXB9-2is significantly higher than that of HOXB9-1 in skin, small intestine, and abdominal fat tissue. HOXB9-2 may play a specific role in the growth of those tissues in black-boned chickens.
HOXB9-encoded nuclear protein functions as a sequence-specific transcription factor that is involved in the proliferation and differentiation of cancer cells (Hayashida et al., 2009; Seki et al., 2012). However, there have been no reports to date regarding the effects of HOXB9 in the pigmentation of animal skin or coats. Lin et al. (2018) reported that HOXB9 can promote melanoma growth and that the protein inhibits cell apoptosis in a mouse xenograft model (Lin et al., 2018). To analyse the potential roles of the two transcripts of HOXB9 in melanin biosynthesis in blackboned chicken skin, the mRNA levels of HOXB9-1 and HOXB9-2 were detected and comparatively analysed in black and white samples. The results showed that HOXB9 was down regulated in black samples, and that the expression of the two transcripts of HOXB9 was significantly different between black and white tissue samples (in feather bulbs and skin). Current evidence suggests that HOXB9-1 and HOXB9-2 may play a role in melanogenesis of the feathers and skin of chickens. These data are also consistent with the RNA sequencing analysis results in our previous study (Yu et al., 2018). Interestingly, HOXB9-1 was undetectable in both black and white breast muscle tissues, whereas the mRNA levels of HOXB9-2 were associated with black and white muscle colours. The results of this study clearly identifyHOXB9 as a pigmentation gene that may be involved in melanin biosynthesis in chicken feathers, skin, and muscle tissue. Further studies are needed to characterise the molecular mechanisms of HOXB9 and its two transcripts in melanogenesis in the chicken.

In conclusion, we report the cloning and functional expression of two alternative splicing variants (HOXB91 and HOXB9-2) of the HOXB9 gene. Our findings demonstrate the significant associations of the mRNA levels of the two variants with melanogenesis in the feather bulb and skin. Only HOXB9-2, which may play a potential role in muscle melanogenesis, was significantly differentially expressed between black and white muscles $(p<0.05)$. These findings provide 
new insight into our understanding of the biochemical characteristics and physiological role of chicken HOXB9.

\section{ACKNOWLEDGEMENTS}

This work was supported by Sichuan Science and Technology Program (2018JY0452). This work was also funded by Scientific Research Plan Projects of Locality and University Cooperation of Leshan Normal University (HX170068), and the Scientific Research Project by Leshan Normal University (Z16020).

\section{REFERENCES}

Bourgeois YXC, Bertrand JAM, Delahaie B, Cornuault J, Duval T, Milá B, et al. Candidate gene analysis suggests untapped genetic complexity in melanin-based pigmentation in birds. Journal of Heredity 2016;107(4):327-335.

Chang CM, Coville JL, Coquerelle G, Gourichon D, Oulmouden A, TixierBoichard M. Complete association between a retroviral insertion in the tyrosinase gene and the recessive white mutation in chickens. BMC Genomics 2006;7(1):19.

Cheah MT, Wachter A, Sudarsan N, Breaker RR. Control of alternative RNA splicing and gene expression by eukaryotic riboswitches. Nature 2007;447(7143):497-500.

Cho $M$, Ryu $M$, Jeong $Y$, Chung $Y-H$, Kim D-E, Cho H-S, et al.

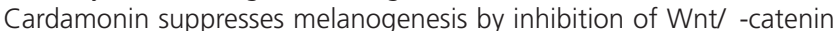
signaling. Biochemical and biophysical research communications 2009;390(3):500-505.

Cieslak M, Reissmann M, Hofreiter M, Ludwig A. Colours of domestication. Biological Reviews of the Cambridge Philosophical Society 2011;86(4):885-899.

Dorshorst B, Molin AM, Rubin CJ, Johansson AM, Strömstedt L, Pham MH, et al. A complex genomic rearrangement involving the endothelin 3 locus causes dermal hyperpigmentation in the chicken. PLoS Genetics 2011;7(12):e1002412.

Faraco CD, Vaz SAS, Pástor MVD, Erickson CA. Hyperpigmentation in the Silkie fowl correlates with abnormal migration of faterestricted melanoblasts and loss of environmental barrier molecules. Developmental Dynamics An Official Publication of the American Association of Anatomists 2001;220(3):212-225.

Hayashida T, Takahashi F, Chiba N, Brachtel E, Jinno H, Kitagawa Y, et al. HOXB9 promotes the acquisition of tumorigenic phenotypes in mammary epithelial cells. Cancer Research 2009;69(24):6145.

Hume AN, Ushakov DS, Tarafder AK, Ferenczi MA, Seabra MC. Rab27a and MyoVa are the primary Mlph interactors regulating melanosome transport in melanocytes. Journal of Cell Science 2007;120(Pt 17):3111-3122.

Kerje S, Sharma PU, Kim H, Bagchi S, Fredriksson R, Schutz K, et al. The Dominant white, Dun and Smoky color variants in chicken are associated with insertion/deletion polymorphisms in the PMEL17 gene. Genetics 2004;168(3):1507-1518.

Lin J, Zhang D, Fan Y, Chao Y, Chang J, Li N, et al. Regulation of cancer stem cell self-renewal by HOXB9 antagonizes endoplasmic reticulum stressinduced melanoma cell apoptosis via the miR-765-FOXA2 Axis. Journal of Investigative Dermatology 2018;138(7):1609-1619.
Lu ZX, Huang Q, Park JW, Shen S, Lin L, Tokheim CJ, et al. Transcriptomewide landscape of pre-mRNA alternative splicing associated with metastatic colonization. Molecular Cancer Research 2015;13(2):305318.

Mcguire AM, Pearson MD, Neafsey DE, Galagan JE. Cross-kingdom patterns of alternative splicing and splice recognition. Genome Biology 2008;9(3):R50.

Mudge JM, Frankish A, Fernandezbanet J, Alioto T, Derrien T, Howald C, et al. The origins, evolution, and functional potential of alternative splicing in vertebrates. Molecular Biology and Evolution 2011;28(10):29492959.

Nguyen DX, Chiang AC, Zhang XH, Kim JY, Kris MG, Ladanyi M, et al. WNT/ TCF signaling through LEF1 and HOXB9 mediates lung adenocarcinoma metastasis. Cell 2009;138(1):51-62.

Pan Q, Shai O, Lee LJ, Frey BJ, Blencowe BJ. Deep surveying of alternative splicing complexity in the human transcriptome by high-throughput sequencing. Nature Genetics 2008;40(12):1413-1415.

Seki H, Hayashida T, Jinno H, Hirose S, Sakata M, Takahashi M, et al. HOXB9 expression promoting tumor cell proliferation and angiogenesis is associated with clinical outcomes in breast cancer patients. Annals of Surgical Oncology 2012;19(6):1831-1840.

Simpson CG, Manthri S, Raczynska KD, Kalyna M, Lewandowska D, Kusenda $B$, et al. Regulation of plant gene expression by alternative splicing. Biochemical Society Transactions 2010;38(2):667-671.

Thatcher SR, Zhou W, Leonard A, Wang BB, Beatty M, Zastrowhayes G, et al. Genome-wide analysis of alternative splicing in Zea mays: landscape and genetic regulation. Plant Cell 2014;26(9):3472.

Tian Y, Xie M, Wang W, Wu H, Fu Z, Lin L. Determination of carnosine in Black-bonedSilky Fowl (Gallus gallus domesticus Brisson) and common chicken by HPLC. European Food Research \& Technology 226(1):31114.

Voisey J, Van DA. Agouti: from mouse to man, from skin to fat. Pigment Cell Research 2002;15(1):10-18.

Widelitz RB. Wht signaling in skin organogenesis. Organogenesis 2008;4(2):123-133

Yamaguchi Y, Passeron T, Watabe H, Yasumoto K, Rouzaud F, Hoashi T, et al. The effects of dickkopf 1 on gene expression and Wnt signaling by melanocytes: mechanisms underlying its suppression of melanocyte function and proliferation. Journal of Investigative Dermatology 2007:127(5):1217-1225.

Yu S, Liao J, Tang M, Wang $Y$, Wei $X$, Mao L, et al. A functional single nucleotide polymorphism in the tyrosinase gene promoter affects skin color and transcription activity in the black-boned chicken. Poultry Science 2017:96(11):4061-4067.

Yu S, Wang G, Liao J, Tang M, Sun W. Transcriptome profile analysis of mechanisms of black and white plumage determination in black-boned chicken. Cellular Physiology and Biochemistry 2018;46(6):2373-2384.

Yamaguchi Y, Itami S, Watabe H, Yasumoto K, Abdel-Malek ZA, Kubo $T$, et al. Mesenchymal-epithelial interactions in the skin: increased expression of dickkopf1 by palmoplantar fibroblasts inhibits melanocyte growth and differentiation. Journal of Cell Biology 2004;165(2):275285.

Zhang J, Liu F, Cao J, Liu X. Skin transcriptome profiles associated with skin color in chickens. PLoS One 2015;10(6):e0127301.

Zhang XD, Wang HH, Zhang CX, Li QH, Chen XH, Lou LF. Analysis of skin color change and related gene expression after crossing of Dongxiang black chicken and ISA layer. Genetics and Molecular Research 2015;14(3):11551-11561 